\title{
Horizons/Théâtre
}

Revue d'études théâtrales

12 | 2018

Les dramaturgies arabes et l'Occident

\section{Au-delà du voile de Slimane Benaïssa : de la mise en parole arabe à la mise en scène occidentale}

Janice Gross

\section{(2) OpenEdition}

Journals

Édition électronique

URL : http://journals.openedition.org/ht/408

DOI : $10.4000 /$ ht.408

ISSN : 2678-5420

Éditeur

Presses universitaires de Bordeaux

Édition imprimée

Date de publication : 1 janvier 2018

Pagination : 110-128

ISSN : 2261-4591

\section{Référence électronique}

Janice Gross, «Au-delà du voile de Slimane Benaïssa : de la mise en parole arabe à la mise en scène occidentale», Horizons/Théâtre [En ligne], 12 | 2018, mis en ligne le 01 janvier 2019, consulté le 21 juillet 2019. URL : http://journals.openedition.org/ht/408 ; DOI : 10.4000/ht.408

\section{(c) $(1)$}

La revue Horizons/Théâtre est mise à disposition selon les termes de la Licence Creative Commons Attribution - Pas d'Utilisation Commerciale - Pas de Modification 4.0 International. 


\section{JANICE GROSS}

Janice Gross est professeur émérite. Elle a enseigné les langues modernes: français et arabe au Grinnell College, Grinnell (lowa) USA. Ell est la traductrice en anglais de La dernière nuit d'un damné de Slimane Benaïssa, The Last Night of a Damned Soul (Grove Press, 2004). Elle a également publié articles, entretiens, et essais: " Au-delà du tragique: une traversée théâtrale - entretien avec Slimane Benaïssa » (2014); "Performing Beyond Trauma: Stages of Recovery in Slimane Benaïssa's Theatre "; "Albert Camus and Contemporary Algerian Playwrights: A Shared Faith in Dialogue "; "France and Algeria: "Performing the Impossible Memory" of a Shared Past »; " Abd el-Kader: Celebrating a Muslim Hero in lowa ».

Mail : grossj@grinnell.edu

Résumé: Slimane Benaïssa, figure de prove du théâtre algérien depuis cinq décennies, a créé Rak khouya, ou ana chkoun? [Si tu es mon frère, qui suis-je?] en 1990, sa dernière pièce en arabe avant son exil. Succès à scandale de courte durée en Algérie, cette pièce serait la seule à être traduite en français par l'auteur. Mettant en scène le vif débat entre deux sœurs ayant des points de vues opposées à propos du port du hidjab, Au-delà du voile (Lansman 1991) s'exilerait tout comme son auteur passerelle se détournant de l'arabe dialectal pour s'ouvrir à de nouveaux publics francophones. La pièce offre un cas d'étude du processus d'adapta-

Abstract: Leading figure of the Algerian stage for five decades, Slimane Benaïssa created Rak khouya, ou ana chkoun? [If you are my brother, then who am I?] in 1990. A succès de scandale cut short in Algeria, it would be the last play of his Arab repertoire before his exile and the only one that he would translate into French. Au-delà du voile [Beyond the Veil] (Lansman, 1991) performs a lively debate between two sisters arguing opposing viewpoints on a woman's right to refuse the hijab (veil). The play's exile and adaptation to the French stage mirror Benaïssa's own transition between Algeria and dialectical Arabic and new Frenchspeaking audiences. Au-delà du voile provides a case study of a process of scenic tion où s'affrontent et s'échangent langues, histoires, cultures, dramaturgies, et publics. Mise en scène par Agnès Renaud (20042014), cette " pièce voyageuse " donne à approfondir la problématique interculturelle de "l'intraduisible» de Rak khouya ou ana choukn? sur la scène occidentale et à interroger les raisons pour lesquelles Au-delà du voile reste toujours d'actualité.

Mots-clés: adaptation scénique, Algérie, arabe (dialectal) algérien, exil, femmes musulmanes, France (français), hidjab (voile), interculturel, Islam (musulman), traduction

adaptation at the intersection of language, national histories, cultures, stage practices, and audiences. Based on French director Agnès Renaud's scenic adaptation (20042014), this study explores the intercultural challenges posed by the "untranslatable » aspects of performing Rak khouya, ou ana chkoun? to French audiences, and considers what accounts for Au-delà du voile's continued relevance.

Keywords: Algeria, dialectal Arabic (Algerian Arabic), exile, France (French), hijab (veil), intercultural, Islam (Muslim), Muslim women, stage adaptation, translation 


\section{Au-delà du voile de Slimane Benaïssa : de la mise en parole arabe à la mise en scène occidentale}

Là où la carte découpe, le récit traverse. Il est « diégèse », dit le grec pour désigner la narration : il instaure une marche (il « guide $\gg)$ et il passe à travers (il « transgresse $\gg)$.

Michel de Certeau 189

Slimane Benaïssa, Figure De proue du théÂtre AlGÉRIEN populaire drainait le grand public en Algérie pendant des années 1970 et 1980. Cependant, son théâtre, lieu de contestation devenu culte, et sa voix éponyme d'arabe algérien prennent fin (du moins provisoirement ${ }^{1}$ ) avec Rak khouya ou ana chkoun? [Si tu es mon frère, moi qui suis-je?] en 1991. Son dernier spectacle, créé en arabe algérien, marque une transition entre la période algérienne et l'exil de l'artiste, qui a été obligé, comme tant d'autres, de quitter l'Algérie à cause des violences de la décennie noire. Mais, avec Rak khouya ou ana chkoun? Benaïssa laisse un dernier mot urgent à son public fidèle. À partir des prises de position entre deux sœurs au sujet du hidjab, le dramaturge propose « une argumentation mise aux services des gens pour qu'ils se défendent » contre les intégristes ${ }^{2}$. Pièce de transition pour le dramaturge-acteur, pour son pays, et pour sa pratique scénique arabe, Rak khouya exprime l'esprit vif et incisif du dramaturge qui « dévoile le mieux ces nombreux conflits et abcès, noués et cachés [...] qu'on aimerait plutôt maintenir occultés $\gg$ (Déjeux: 280). Mais, c'est aussi une pièce qui annonce la « scène obscure »d'Edward Bond: « scène du portail, du seuil [...] où vous êtes devant un choix ${ }^{3}$ ». Lors du questionnement identitaire du titre ( $\mathrm{Si}$ tu es mon frère, moi qui suis-je ?), Rak khouya engage personnages, dramaturge, et citoyens algériens à affronter leur avenir inconnu « aux portes de l'Histoire ». D'autant plus signifiant, cette dernière pièce en arabe, tout comme le parcours de son auteur, mettra en marche une traversée transnationale reliant la création intraculturelle conçue pour le public algérien et un engagement interculturel « au-delà »de l'Algérie, comme le suggère son titre en traduction. 


\section{Mise en scène de la parole plurielle de la femme}

Donner voix à des protagonistes femmes, à deux sœurs adultes débattant en toute franchise du port du hidjab que leur frère impose à la sœur cadette rebelle marque un nouveau tournant à la fois thématique et scénique chez Benaïssa. En plus de rompre avec la pratique théâtrale arabe (et avec la sienne d'ailleurs) qui laissait peu de place aux femmes (Cheniki 51), cette nouvelle approche « plus intimiste » met en scène la voix opprimée (et plurielle) des Algériennes au quotidien où « l'individu seul se trouve en proie aux contradictions de la société $\gg$. Pièce de passage, elle se situe entre contestation et émancipation, entre critiquer le parti unique FLN (Front national de libération) et démasquer les islamistes, et faire réfléchir le public et le « responsabiliser $\gg$ (Entretien, 159).

Dans un nouveau paradigme plus dialogique que dialectique, la stratégie de la parole permet aux deux sœurs de s'engager et se disputer dans un «nouvel espace » de liberté individuelle où elles se confrontent l'une à l'autre à travers des échanges mêlant politique et poétique, attaques et ententes, jeux, chants et réflexions. Porteuses de diverses perspectives (mariée/célibataire; sans études/instruite; traditionnelle/moderne; soumise/rebelle; voilée/ non voilée), les sœurs s'approprient un nouvel espace dialogique grâce auquel elles arrivent à se dire et se découvrir. Rak khouya, véritable récit qui «traverse » un terrain inconnu au sens décrit par Certeau, s'inscrit dans un processus théâtral qui « guide » et « transgresse » simultanément. À travers le combat entre les sœurs, le récit « guide » le spectateur vers des connaissances vraies et fiables sur l'histoire du pays, de l'islam et du hidjab, alors qu'il transgresse les limites et brise les tabous pour les dévoiler. Par exemple, le beau discours du martyr mystique Al-Hallaj sur l'amour de Dieu se juxtapose à l'image de l'Islam du frère: « Ce qui manque à l'Islam de mon frère, c'est la sincérité, l'amour et la sérénité dans la paix. Dieu nous a donné un Islam pour vivre. Eux, ils ont inventé quatorze Islams pour mourir » (17-18)4. La théâtralité de ses oppositions prend toute son ampleur scénique avec chants, monologues intérieurs, voix off, argumentations, scènes de mime et de caricature des hommes au pouvoir, et le rituel ancestral de la bouqqala ${ }^{5}$ détourné en parodie politique. Pour le public algérien, la pièce « était d'une vérité fulgurante $[\ldots]$ il y a eu des bagarres sérieuses dans les familles $\gg$ (Entretien, Gross, 160). 
Sous-tendue par un questionnement identitaire, une nouvelle démarche dialogique s'annonce : l'ouverture à autrui. Alors que dans d'autres pièces, les personnages exclusivement mâles restent isolés et opposés dans leur propre espace idéologique de combat, les deux sœurs se disputent en s'écoutant, s'engageant dans une démarche proprement intersubjective et intraculturelle. C'est à travers le regard intérieur de la femme en tant qu' individu que se dévoile la crise sociopolitique extérieure. Éloignées des contraintes sociales, elles se rencontrent dans l'entre-deux : à mi-chemin entre altérité et solidarité où le sens de l'autre s'impose et se fait valoir. Une stratégie de la parole traversée par le dialogique en combat et le monologique en réflexion, une énonciation où s'entrecroisent la critique sociale et la remise en question du moi. De même, les sœurs en débat incarnent deux Algéries en train de se confronter à l'actualité politique de leur pays. Pour les spectateurs, cela servira de modèle pour « se défendre » contre le spectre islamiste. En fait, c'est la femme enfermée et réduite au silence qui y voit clair : « Les hommes croient nous avoir donné la liberté. Ce qu'ils ne savent pas, c'est que leur liberté dépend de tellement de choses; en réalité ils partagent avec nous leur dépendance $\gg(5)$. De même, la pièce fait écho au drame du dramaturge dans le dilemme énoncé par la sœur cadette, cette Antigone, à la fin de la pièce : « Dois-je [...] engager des combats impossibles? Dois-je pour exister, choisir l'exil de l'exil ? » (35).

\section{Au-delà du voile / de l'Algérie}

Bien que la spécificité culturelle du conflit initial de la pièce soit axée sur la problématique du hidjab, l'énonciation narrative s'étend au-delà de la simple polémique d'être pour ou contre:

L'aînée: Ce qui arrive entre toi et ton frère, ce n'est pas qu'une affaire de hidjab.... Je ne peux le croire.

La cadette: Tu as raison, ce n'est pas uniquement une affaire de hidjab. C'est une question de liberté et de tolérance: je veux décider librement de mes actes (28).

Ainsi, une problématique spécifique à la femme musulmane ouvre la voie à une autre plus grande interrogation d'ordre philosophique : la liberté de l'individu face au choix. Superposant la crise existentielle du « dedans » à la plus grande crise sociopolitique et religieuse, celle qui menace tout Algérien « du dehors $\gg$, le récit de la pièce permet aux spectateurs arabes de témoigner «simultanément de l'événement et de l'intimité du sujet ${ }^{6} \gg$. Parole occultée et réprimée de la femme musulmane qui ose dire, sentir, rire et raisonner, elle prépare un parcours théâtral qui se projettera bien au-delà du vécu algérien. 
Point tournant dramaturgique, Rak khouya ou ana chkoun? (Au-delà du voile) initie une traversée entre les deux rives, lançant la « destinée voyageuse $^{7} \gg$ de Benaïssa, passeur interculturel, sur les planches occidentales et auprès des publics francophones et autres ${ }^{8}$. À la suite d'un succès théâtral de courte durée en 1991, la pièce, d'emblée controversée, diffusée sur sur ENTV, sera retirée rapidement de l'affiche dans une Algérie au seuil des violences des Années noires. Rak khouya ne sera désormais jamais repris sur la scène arabe. Pourtant, une fois traduite d'urgence en français par l'auteur et rebaptisée Au-delà du voile (Éditions Lansman 1991), la pièce se jouera d'abord au Festival International des Francophonies en Limousin et effectuera une tournée (1992). Souvent jouée en deux langues en alternance par les mêmes actrices algériennes, la pièce aura 42 représentations 9.

Itinéraire :

\begin{tabular}{|l|l|}
\hline 1991 & Algérie Rak khouya ou ana chkoun (mise en scène de Benaïssa) \\
\hline $1991-1992$ & $\begin{array}{l}8^{\mathrm{e}} \text { Festival des Francophonies en Limousin } \\
(\text { Au-delà du voile et Rak khouya ou ana chkoun - joué en deux langues) }\end{array}$ \\
\hline 1991 & Éditions Lansman ( $1^{\text {re }}$ éd. $)$ \\
\hline 2004 & Mise en scène d'Agnès de Renaud \\
\hline 2008 & Éditions Lansman $\left(2^{\mathrm{e}}\right.$ éd. $)$ \\
\hline 2008 & Avignon (mise en scène de Renaud) \\
\hline $2010-2011$ & Paris (Lucernaire) (mise en scène de Renaud) \\
\hline 2011 & Émission du Public Sénat - Benaïssa et des actrices ${ }^{\mathbf{1 0}}$ \\
\hline 2014 & Dernière représentation (mise en scène de Renaud) \\
\hline
\end{tabular}

\section{S'engager ailleurs et autrement}

Seule pièce de Benaïssa à être traduite par l'auteur lui-même et à être adaptée sur scène par un tiers, Au-delà $d u$ voile, choisie par Agnès Renaud et la Compagnie de l'Arcade (de la Forge), se jouera sur les scènes européennes de 2004 à 2014: notamment à Avignon (2008) et à Paris au Lucernaire en 2011 où la critique attira l'attention sur sa capacité de ne pas avoir pris « une ride ». C'est à partir d'un tel propos que nous nous interrogerons sur les enjeux associés à ce passage transnational et interculturel et les raisons pour lesquelles $A u$-delà du voile a pu rester d'actualité pendant une vingtaine d'années. Lors de sa production au Lucernaire en 2011, l'émission culturelle « Paroles du monde » du Public Sénat réunira Benaïssa et les deux comédiennes pour discuter de la pertinence actuelle de la pièce. Cet échange télévisé illustre le sens du mot interculturel où « le préfixe inter [...] implique une mise en relation et 
une prise en considération des interactions entre des groupes, des individus, des identités $\gg$ (Abdullah-Pretceille : L'éducation 49). Le message porteur de révolte de la pièce résonnait d'autant plus à la lumière du printemps arabe qui vient d'éclater ${ }^{11}$. Pour les actrices et l'auteur, la signification actuelle s'appuyait sur deux processus significatifs : la révolte (au sens large du terme) au sein de la famille et de la société, comme celle de la jeune génération du monde arabe, et le choix de se confronter aux voiles « intérieurs » en poursuivant ce que l'auteur avait créé comme un « cheminement intérieur jusqu'au bout » pour s'en libérer. Cet échange hors du théâtre entre artistes, auteur et public illustre le rôle critique d'un engagement d'échange que pratique Benaïssa depuis des décennies. Dwight Conquergood le définit en termes anthropologiques comme une performance dialogique dont le but est de « faire rencontrer le moi et l'autre pour qu'ils interrogent, débattent, et s'interrogent l'un l'autre. C'est le genre de performance qui résiste aux conclusions. [...] Elle rapproche le moi et l'autre tout en les empêchant de se joindre. Plus comme un trait d'union qu'un point ${ }^{12} \gg$ [notre traduction].

Il conviendra donc d'examiner ce «trait d'union » à la fois linguistique et scénique qui relie Rak khouya ou ana chkoun à $A u$-delà du voile tout en les gardant séparés. Il s'agira de mettre en contraste deux représentations de la pièce séparées dans le temps et l'espace: celle de Benaïssa télévisée en 1991 en arabe algérien et celle de l'adaptation et mise en scène d'Agnès Renaud et la Compagnie de l'Arcade, au théâtre Lucernaire à Paris en $2011^{13}$. Afin de les comparer en termes de production et de réception, on s'appuiera sur la notion de niveaux de « lisibilité » définis par Patrice Pavis, notamment narrative (la compréhension de la fable et sa structure textuelle), idéologique («dramaturgique $\gg$ à mon sens, projet de la mise en scène), culturelle (le référentiel du monde représenté), et globale ${ }^{14}$ (Analyse, 258-260). Certes, « la lisibilité culturelle » est la plus variable et prend forme comme « le produit d'une construction qui résulte d'une chaîne de lisibilités spécifiques et partielles : lisibilité formelle, narrative ou anti-narrative, idéologique [...] $\gg$ (Pavis Analyse 258). À l'exemple de $A u$-delà du voile, toute création de Benaïssa offre une « lisibilité » narrative qui vise à faire réfléchir, rire, et sentir un public populaire et divers. Équilibriste d'idées, Benaïssa maîtrise l'art d'agencer des prises de positions opposées avec justesse, complexité, humour et ambiguité jouant sur ce que R. Baffet appelle « la stratégie de la corde raide ${ }^{15} \gg$. En présentant au spectateur « une lucidité idéologique »à la fois spécifique au à un contexte précis et d'une portée universelle, la lisibilité narrative de l'œuvre de Benaïssa la rend transposable, voire fait d'elle un trait d'union « interculturel ». 
Pourtant, au niveau de la lisibilité culturelle dans la production et la réception, il faut affronter un dilemme interculturel et faire face à deux « tentations »: «soit donner une vision universelle, voire universaliste de l'être humain [...] ; soit, au contraire, insister sur le particularisme de toute culture... » (Pavis Dictionnaire 132). La recherche d'un équilibre entre ces deux forces exprime bien les enjeux d'une telle transposition dans la mise en scène proposée par Renaud. Certes, de nombreux sujets d'actualité générale se présentent: la répression à l'égard des femmes, l'individu en révolte, et bien sûr, l'islam, l'intégrisme et le port du voile. D'autant plus dans le cas de ce dernier, où la problématique de la « liberté » de choix dans le régime public-privé s'avère compliquée et même dissonante ${ }^{16}$ une fois transférée à l'espace français e (la loi sur la laïcité en 2004 et contestation plus récente contre l'interdiction du port du voile « pas d'atteinte à ma liberté »). En dépit des attitudes en mutation envers le voile, la lisibilité narrative de la pièce ne tranche pas la question du pour ou contre; c'est la question du choix qui prime. Le fait que la sœur aînée choisit de défendre la liberté de la cadette contre leur frère au dénouement de la pièce ne change rien en ce qui concerne sa propre décision de porter le hidjab.

Face à ce dilemme (spécificité culturelle / portée générale), le projet scénique de Renaud vise à accentuer la portée universelle de l'altérité de l'Autre: « Pour moi, ce texte dépasse le cadre de l'Algérie. Il parle de choses qui touchent tout le monde. [...] Je pense que les Occidentaux peuvent se retrouver dans cette pièce qui permet de créer des débats bien sûr sur le voile, mais aussi entre les communautés diverses ${ }^{17}$. $\gg$ De même, la voix des femmes conçue par Benaïssa se croise avec une autre thématique chère à Renaud: celle de la femme dont la parole «se cherche, se heurte et tente de dire. Un verbe inquiet, en conflit, insatisfait ${ }^{18} \gg$. En dépit d'une résonance universalisante de la pièce, la complexité socioculturelle et politico-religieuse de l'Algérie lors des années 1990 amène Renaud et sa Compagnie à un travail extra-scénique d'appui dont un Dossier pédagogique pour des ateliers et visites d'écoles.

Bien que l'énonciation narrative soit transmissible à tous les publics, rendre la pièce lisibile dramaturgiquement et idéologiquement pour un public occidental et non musulman pose certaines difficultés du point de vue du transfert socioculturel. D'une part, il s'agit d'éviter une spécificité excessive et la généralisation abusive, surtout au regard du voile, sujet déjà fortement char- 
gé, sans pour autant dénaturer la spécificité politique du contexte algérien car « ce n'est pas qu'une affaire de hidjab ». D'autre part, il faut transmettre un univers à la fois cohérent et compréhensible dans sa diversité intraculturelle (musulmane, berbère, etc.) conçue en arabe et destinée à un public algérien en proie aux événements de 1990.

\section{Créer au-delà de la langue arabe}

Si on considère que $[\ldots]$ toute la construction vise à rendre les conflits apparents et compréhensibles, on se rend compte que ce qui est universel dans une pièce, c'est le conflit. Le reste est spécifique à la société, et par conséquent à la langue dans laquelle elle est écrite (Slimane Benaïssa) ${ }^{19}$.

Il est clair que l'importance de la langue de création chez Benaïssa est iest capitale. Comment donc arriver à l'universel (le conflit) si on doit se passer de tout ce qui est spécifique à la langue? Le cas du passage entre Rak khouya en arabe algérien et $A u$-delà $d u$ voile en traduction offre un cas d'étude particulièrement révélateur et peu commun : celui de l'auto traduction. L'inventivité enjouée et spontanée en arabe parlé s'affichait comme composante essentielle tout au long d'un spectacle ponctué de petits et gros rires, d'applaudissements, et $d^{\prime}$ « un aspect fête, une communion entre la scène et la salle ». Pour Benaïssa, l'arabe algérien dit « dialectal » représentait la force motrice de son œuvre comme :

1. langue de la contestation par rapport à l'arabe classique

2. langue du théâtre populaire; du peuple pour qui le théâtre reste le lieu où il va pour s'entendre et se comprendre

3. langue de la créativité, d'une poésie qui nourrit le texte

4. langue où chaque personnage «met en œuvre son propre langage, sa rythmique et sa prosodie $\gg$ (Cheniki 84 ).

5. langue de l'humour

6. langue de l'affirmation identitaire; risque d'une « perte de soi » 20.

En tant que traducteur de sa propre pièce pour la première et dernière fois (il en convient), le dramaturge, conscient des pertes occasionnées au cours de ce travail, regrette le résultat d'une traduction opérée dans l'urgence. Désormais, la réécriture remplacera la traduction pour éviter la tension entre une perte de soi et le désir de rencontrer autrui. De l'autre côté, ce «métis heureux » trouvera sa récompense dans la « libération » qu'offre la langue française. Bien que la langue arabe constitue la substantifique moelle de sa 
création, la langue française a aussi sa place dans la mosaïque de son identité hybride : «si l'arabe algérien et le berbère sont à défendre, la langue française en tant qu' héritage vivant chez nous n'est pas à abandonner $\gg$. En plus d'assumer l'exil comme « une école de dépassement de soi », sa vocation comme homme de théâtre de langue française lui permet d'aller à la rencontre de nouveaux publics occidentaux où sa voix comme témoin musulman sera entendue.

\section{Afficher la langue : mettre en scène la parole arabe}

Un regard sur les affiches créées pour les deux productions met en contraste la façon dont chaque spectacle présente son rapport à la parole et au jeu scénique. D'une pratique théâtrale enracinée dans la langue, l'affiche pour Rak khouya ou ana chkoun met en place l'essence proéminente et la force esthétique de l'arabe. Une des dernières œuvres du grand artiste peintre « du signe » Mohammed Khadda, mort en 1991, cette affiche et le décor de Rak khouya expriment une identité ancrée dans sa langue.

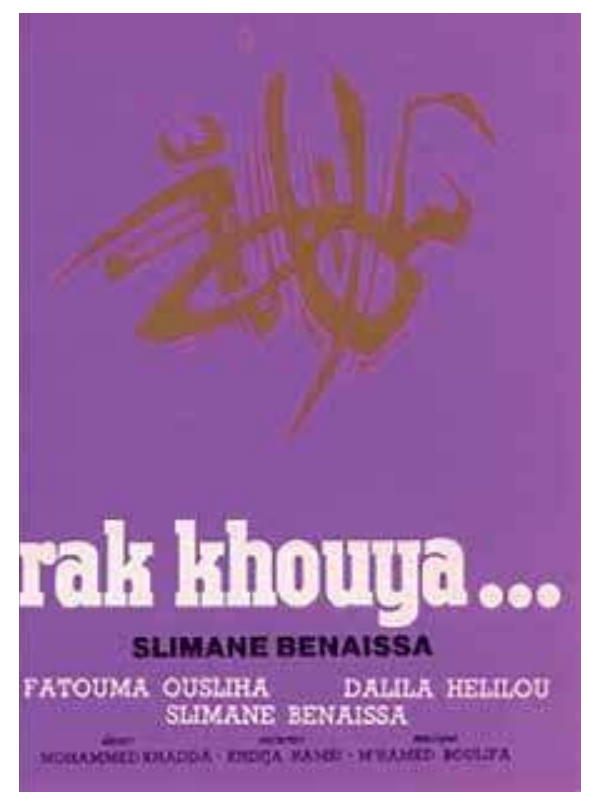

Figure 1 : Affiche en français de Rak khouya ou ana chkoun par Mohammed Khadda, 1991.

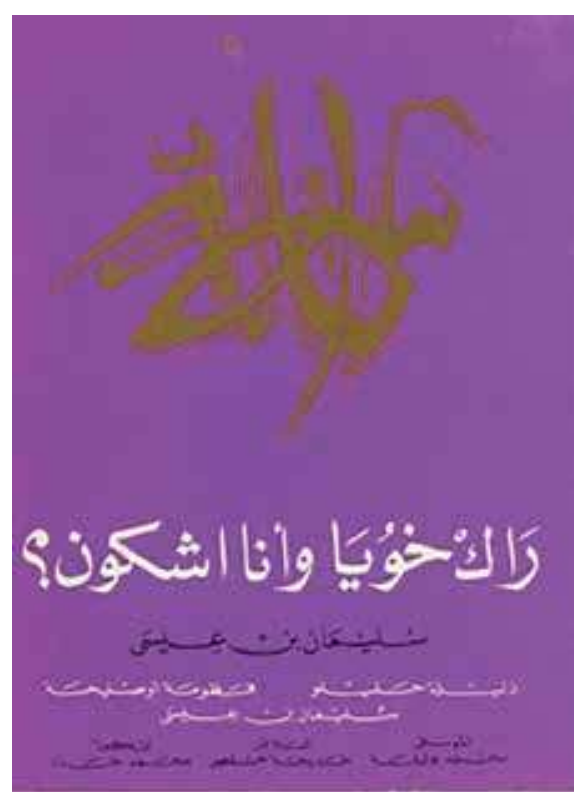

Figure 2: Affiche en arabe de Rak khouya ou ana chkoun par Mohammed Khadda, 1991. 


\section{Afficher la mise en corps}

Destinée au spectateur occidental, l'affiche de Au-delà du voile du Lucernaire repose sur la présentation et la stylisation d'une femme voilée. À la différence de l'affiche théâtrale de l'artiste Khadda inscrite dans la calligraphie arabe, un regard proprement corporel annonce un spectacle où le jeu emporte sur la parole. En plus de deux références au «voile» (dans le titre et la photo d'une femme voilée), l'image forte de la femme en désarroi signale un état d'âme chargé d'émotivité dont le cri étouffé rappelle celui de la Mère courage de Brecht. Sur la scène, cette même image prendra vie dans le personnage de la sœur aînée outrée mais

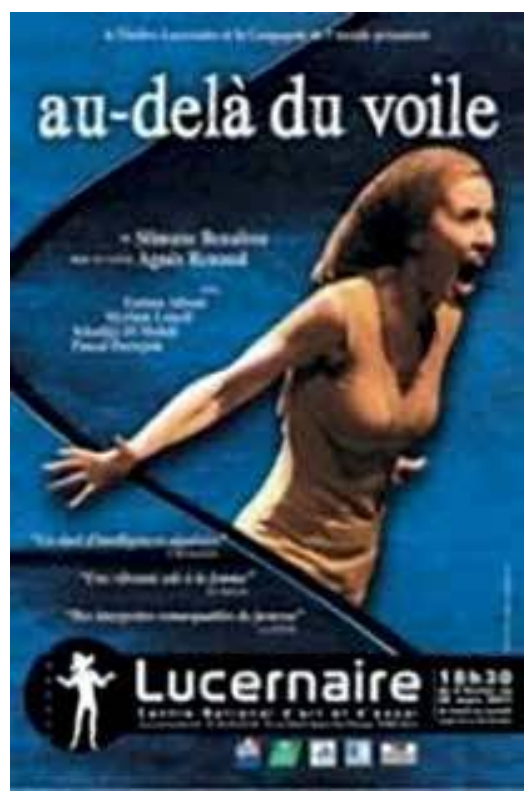

Figure 3: Affiche de Au-delà du voile 2011. incapable de se révolter, combattante véhémente en dispute avec sa sœur cadette mais résignée à se taire sans oser agir. D’un jeu distancié en débat, la colère et la prise de position les enferment chacune dans une altérité à la fois gestuelle et spatiale.

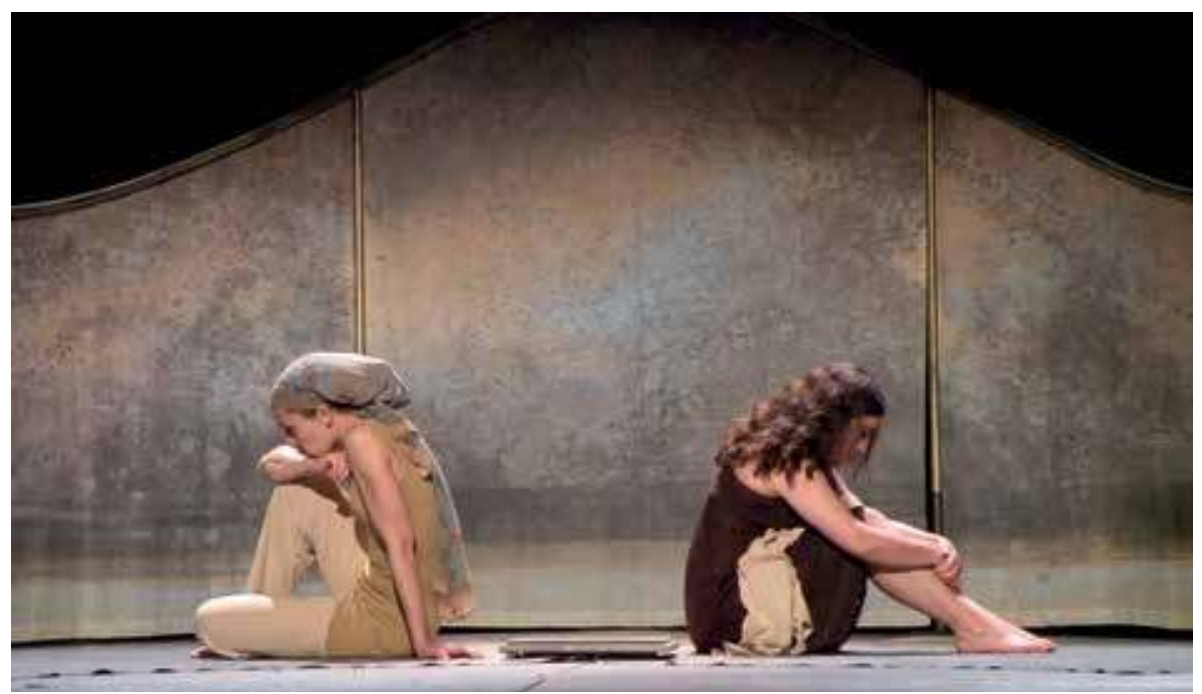

Figure 4 : Entre deux sœurs. 
Deux espaces identitaires, deux altérités ; un même combat: mais la mise en scène en arabe de Benaïssa (1990) et celle de Renaud (2011) soulignent des projets dramaturgiques nettement distincts. Le décor mimétique de Rak khouya plonge le spectateur arabe dans l'espace repérable d'un intérieur familial algérien où les sœurs se parlent et se disputent avec force et conviction dans leur quotidien. Par contre, le décor chez Renaud, renonçant à rechercher un « effet d'authentique » algérien, situe l'échange dans un espace vide et dépouillé de toute décoration avec l'accent mis sur la corporalité, la gestuelle, et la stylisation théâtrale des échanges. Alors que le jeu des actrices en arabe est d'un naturel expressif à la fois enjoué et combatif faisant rire et réagir les spectateurs à de nombreuses références bien familières, le jeu scénique français met l'accent plutôt sur l'isolement des sœurs qui ne se parlent pas face à face mais s'adressent aux spectateurs-juges comme si elles témoignaient devant un tribunal, d'un ton sérieux ponctué seulement de petits moments empreints d'ironie verbale.

La problématique de lisibilité culturelle s'exprime très clairement aussi dans la représentation du voile comme habit: entre le matériel et le symbolique. Une fois rentrée du marché, l'aînée enlève son hidjab (selon la coutume) pour rester non voilée tout au long du spectacle arabe. L’acte de débattre de la question du hidjab (accepter un « abri $\gg$ ou refuser un « linceul $\gg)$ se déroule entre les deux sœurs non voilées bien que la sœur aînée chez Renaud reste voilée. Alors que les deux femmes sans hidjab se parlent aisément en tête à tête, l'aînée restant voilée dans la production occidentale ne sert qu’à renforcer sa prise de position face à la cadette aux cheveux libres. En plus d'être divisées par un dispositif spatial où chacune s'inscrit dans son propre espace, la distinction vestimentaire (voilée/non voilée) souligne davantage le rapport oppositionnel et distancié entre les sœurs, surtout auprès des spectateurs occidentaux pour qui le voile constitue un fort marqueur identitaire. Certes, une représentation « authentique » d'une culture étrangère n'est ni raisonnable ni possible ${ }^{21}$. L'énonciation scénique de Renaud se tourne plutôt vers une recherche de l'authenticité du moi et du for intérieur de chaque sœur dans l'acte d'échanger l'une avec l'autre. Entre se taire et dire sa révolte, et entre le voile de tradition qui « protège », quoique peu, et le hidjab « linceul » qui enferme, l'énoncé du texte insiste plus sur la façon de raisonner que sur le choix en tant que tel. Néanmoins, la force visuelle de l'aînée voilée accentue l'isolement psychologique qu'elle éprouve par rapport à la société et à elle-même « Maudit soit ce "Moi" » (30). De même, le spectateur occidental risque d'être influencé par ce que Miriam Cooke appelle « the weight of the 
veil $\gg$, ce poids du voile qui impose des aprioris concernant la femme voilée et ses idées en dialogue (130).

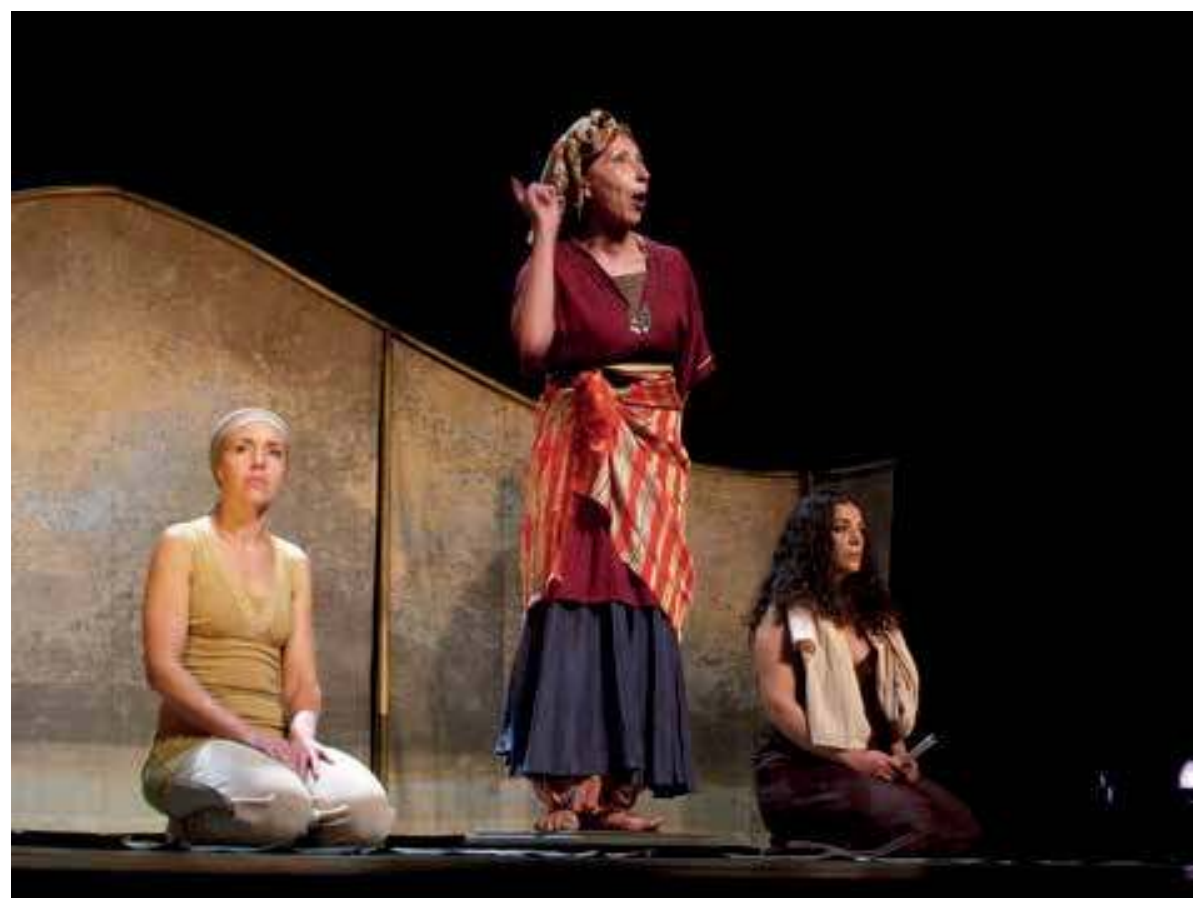

Figure 5 : la $3^{\mathrm{e}}$ voix.

\section{La troisième voix}

Transférer $A u$-delà $d u$ voile à la scène francophone présente un autre défi relié à l'oralité de l'arabe populaire : celui du « grain de la voix » (Barthes) inhérent à l'identité de chaque comédienne, en particulier celle de la sœur aînée qui s'adonne aux chants et aux lamentations lyriques. Pour saisir l'expressivité de l'arabe avec sa ponctuation verbale et gestuelle, Renaud introduit un « troisième regard » pour remplacer la présence vocale arabe représentée par l'aînée. Chanteuse arabophone ${ }^{22}$ à fonctions multiples, ce troisième personnage féminin sert de lien à la langue et à la mémoire ancestrale : « voix d'une autre génération absente, celle de la "Mère" défunte des sœurs orphelines, d'une figure féminine ancestrale $[. .$.$] un personnage porteur de sagesse popu-$ laire, libre de ces déplacements ${ }^{23} \gg$. À la différence de l'expressivité authentique de la sœur aînée qui se donne corps et âme au chant, prolongement de la parole arabe, la troisième présence féminine chez Renaud fonctionne 
comme un médiateur ambulant, un pont à la fois spatial et discursif entre les deux sœurs. Comme une frontière qui « créa la communication autant que la séparation $\gg$ un « espace entre deux $\gg$ (Certeau 187), le tiers arabe de l'Ancêtre (jouant aussi d'autres figures et voix off) fonctionne comme une zone de « passage » scénique entre le réel et l'imaginaire ; l'altérité et la complicité, et le passé et le présent. De même, les interventions sonores d'un percussionniste (Pascal Portejoie) situé derrière un voile transparent renforcent certains changements de ton (douceur/menace). Il est vrai que la mise en scène « apporte une vigueur nouvelle à ce texte $\gg$ des années $1990^{24}$. Pourtant, l'intervention scénique d'un tiers sert aussi à empêcher le rapprochement physique et affectif entre les femmes. Seule présence culturellement codée, l'Ancêtre dans son habit coloré se fait remarquer dans sa spécificité surtout en contraste avec les vêtements neutres à la française des protagonistes, ce que pour certains, pourrait frôler le folklore. Autre modification scénique :

l'Ancêtre (personnage féminin) se déguise en « mufti » prononçant le discours tel que l'imagine la sœur cadette alors que dans la version arabe, le récit sera raconté comme il est de coutume en Algérie par un imam homme, joué en l'occurrence par Benaïssa à la grande joie du public arabe.

\section{Passage ancestral - entre tradition et modernité}

La référence la plus explicite à l'identité socioculturelle algérienne (et facilement jouable), nous la relevons au point culminant de la pièce au moment où les sœurs s'adonnent au jeu inspiré d'un rituel ancestral de divination, la bouqqala. Dans cette performance tirée du «patrimoine » culturel algérien, la bouqqala, « enacts embodied memory » [incarne la mémoire $]^{25}$ pour permettre aux sœurs de se rapprocher dans l'espace liminal de la mémoire partagée et de l'imaginaire où se réunissent la joie d'enfance et la complicité d'adulte. Initiée par l'aînée pour apaiser la sœur désespérée face au destin tragique de l'Islam, la bouqqala ressuscite le côté rêveur et ludique d'un passé ancré dans l'appartenance culturelle et ancestrale momentanément libérée du poids sociopolitique de l'actualité identitaire imposée. Joué en plein «communitas » (Victor Turner), le rituel ludique qui en résulte ranime la force créatrice née au carrefour de l'esprit moderne de la cadette et l'adhérence à la tradition de l'aînée. Ainsi, l'invocation sincère au nom de Dieu « Ouvre-moi une issue dans cette impasse » de l'aînée se transforme en une devinette hilarante des partis politiques par la cadette. Ce jeu de créativité carnavalesque à la Bakhtine offre une scène riche en théâtralité (fumigation, encens) et en solidarité entre les deux sœurs. Pourtant, dans le spectacle français, c'est la troisième femme jouant à l'Ancêtre qui s'impose entre les deux sœurs comme 
meneur du jeu. En arabe, ce rituel parodique plein de références à la politique algérienne a suscité beaucoup de rires chez les Algériens en 1991, alors qu'en français, l'effet scénique s'avère moins efficace faute de références facilement repérables.

Bien que la lisibilité culturelle en arabe ne ne soit pas rendue en français, la lisibilité narrative et idéologique de cette scène de retrouvailles dépend du jeu pour réconcilier les sœurs et laisse place aux « restorative energies ${ }^{26} \gg$, ces énergies capables de rétablir même ce qui semble irréparable (13). Au dénouement, chaque sœur finit par se dévoiler face à l'opposant: l'aînée choisit de défendre la cadette contre le frère ( $\ll$ Si l'un des deux doit sortir de la maison, c'est lui et non toi. C'est à lui de sortir » (34) et la cadette se laisse éprouver la force et la beauté de la tradition. Ainsi, la lisibilité idéologique amène à une « issue » narrative, quoique provisoire, de l'effet réparateur de l'ouverture dialogique à autrui.

\section{Entre altérité et solidarité : jeux d'espaces}

Scène algérienne

\section{Scène française}

\begin{tabular}{|l|l|}
\hline La parole l'emporte sur le jeu & $\begin{array}{l}\text { Le jeu l'emporte sur la parole } \\
\text { Jeu partagé entre les sœurs }\end{array}$ \\
$\begin{array}{l}\text { Décor intérieur réaliste } \\
\text { Effet d'authentique } \\
\text { Gestuelle mimétique }\end{array}$ & $\begin{array}{l}\text { Décor abstrait } \\
\text { Effet neutre } \\
\text { Gestuelle stylisée }\end{array}$ \\
Sœur aînée non voilée & Sœur aînée voilée \\
Dispositif spatial de rapprochement & Dispositif spatial de séparation \\
Actrices face à face (effet de complicité) & Actrices face au public (effet de tribunal) \\
Chants de l'aînée & $\begin{array}{l}\text { Chants et jeux mimés de la } 3^{\text {e }} \text { femme } \\
\text { Interventions sonores d'un musicien }\end{array}$ \\
\hline Rires de solidarité, de complicité (public) & Rires de compréhension (public) \\
\hline
\end{tabular}

Ayant des objectifs communs, Benaïssa et Renaud répondent au même appel dramaturgique, chacun à sa façon. Pour Renaud elle-même comme pour ses spectateurs de l'Occident, il s'agit d'affronter l'altérité de l'autre pour le rencontrer au-delà des différences : « C'est cette antinomie qui m’intéressait énormé- 
ment. J'avais envie d'aller à la rencontre de l'autre, des autres, de l'Islam aussi, de l'ailleurs ». Il faut dire que la lisibilité narrative du texte (axée sur la liberté de choix) et sa construction idéologiquement équilibrée (ni pour, ni contre le voile) se prêtent bien au transfert « au-delà » de l'Algérie. De même, grâce à sa mise en scène de l'altérité, cette pièce réalise un passage interculturel et transnational qui correspond à la thèse percutante de Martine Pretceille :

Apprendre à voir, à écouter, à être attentif à autrui, apprendre la vigilance et l'ouverture dans une perspective de diversité et non de différences renvoient à la reconnaissance et à l'expérience de l'altérité, expérience qui s'acquiert et se travaille dans la durée ${ }^{27}$.

En plus de donner voix à la femme absente de la vie sociale et de la scène algérienne et musulmane, $A u$-delà $d u$ voile ouvre la voie à la diversité des multi-appartenances de chaque femme dans son individualité.

\section{Citoyen global de la scène mondiale}

Il est indéniable que Slimane Benaïssa est un homme de théâtre algérien, mais il faut bien se rendre compte qu'en dix ans, sans se renier, il est devenu un écrivain et un dramaturge de France [...] au nom de notre mémoire commune.

Dominique Caubet, Discours de présentation de la marraine de Slimane Benaïssa, distingué docteur honoris causa, la Sorbonne, INALCO, 2005

Il est rare qu'un homme de théâtre (auteur, acteur, metteur en scène, auto-traducteur) effectue un parcours francophone et un retour algérien (Wel moudja wellet, juin 2011) reliant langues, cultures, dramaturgies, et publics. Pièce charnière et première création à se faire connaître sur les planches occidentales, Au-delà du voile marque un moment de passage exemplaire. Né du désir inébranlable d'aller à la rencontre d'autrui, ce travail théâtral et sa traduction par l'auteur fournissent un texte capable d'aller « au-delà » de la rive algérienne tout en gardant une certaine résonance de sa spécificité socioculturelle devenue de plus en plus pertinente. Fidèle à une éthique d'engagement, Benaïssa tient au principe que « le théâtre doit permettre au public de multiplier ses expériences pour mieux raisonner une réalité. Il construit la citoyenneté » (Entretien FR 155).

Confier ce rôle critique à la femme musulmane aux multiples subjectivités et expériences, marque un point tournant pour $A u$-delà du voile et sa réso- 
nance globale. Après avoir donné voix à la femme musulmane en arabe, Benaïssa continuera à mettre en scène la femme arabe dans toute sa diversité $^{28}$. Représenter l'expérience plurielle de la femme musulmane aux spectateurs de l'Occident promeut une autre pratique dialogique « au-delà $\mathrm{du}$ voile $»$ : celle que Miriam Cooke appelle un nouveau «Islamic feminism » qui n'est pas une identité fixe, mais fluide, dynamique, et capable de surmonter le poids du voile «the weight of the veil » (59). À partir de leurs appartenances multiples « multiples belongings » et leur engagement double, les deux sœurs illustrent ce que Cooke envisage comme la capacité des femmes musulmanes à s'engager dans une « multiple critique » au sein de leur communauté sans perdre le droit de continuer d'en faire partie intégrante (113).

C'est dans ce même esprit de « partage des voix » (J.-L. Nancy) que les démarches de Benaïssa, comme auteur-traducteur, et de Renaud, comme metteuse en scène, se retrouvent. S'engager dans l'acte périlleux de «traduire », que ce soit linguistique, théâtral, interculturel, ou interhumain, n'est pas sans risque. Mais, avec un effort pour « amener le lecteur [le spectateur] à l'auteur $\gg$ et vice-versa, il y a des chances que l'on découvre ce que Paul Ricoeur appelle « l'hospitalité langagière » où « le plaisir d'habiter la langue de l'autre est compensé par le plaisir de recevoir chez soi, dans sa propre demeure d'accueil, la parole de l'étranger » (20). En fait, le théâtre de Benaïssa, et cette pièce en particulier, offrent aux spectateurs de tous bords un lieu hospitalier où une rencontre socioculturelle mène à une réflexion globale. Rencontre avec l'altérité de la femme musulmane et réflexion sur la lutte universelle pour le droit aux droits. Est-ce peut-être la raison pour laquelle Rak khouya ou ana choukn traduite $\mathrm{Au}$ delà $d u$ voile a pu garder sa pertinence pendant plus d'une vingtaine d'années?

\section{Bibliographie}

Abdallah-Pretceille Martine. L'éducation interculturelle, $2^{\mathrm{e}}$ éd., Paris, Éditions PUF, Collection «Que sais-je? », 2005.

__, "L'interculturel comme paradigme de transgression par rapport au culturalisme ", Voix plurielles, 12.2, 2015, p. 251-263.

Baffet Roseline, «Entre théâtre et roman: Slimane Benaïssa et ses sept lieux d'écriture », Chikhi Beïda (éd.), Destinées voyageuses: la patrie, la France, le monde, Paris, PUPS 2006.

—, Tradition thêâtrale et modernité en Algérie, Paris, L'Harmattan, 1985.

Balibar Étienne, Saeculum, Culture, religion, idéologie, Galilée, 2012. 
Benaïssa Slimane, Au-delà du voile, Carnières-Morlanwelz, Lansman, 1998 [1991]. —-, Marianne et le marabout, Carnières-Morlanwelz, Lansman, 1995.

\section{—, Les fils de l'amertume, Carnières-Morlanwelz, Lansman, 1997 [1996].}

- Un homme ordinaire pour quatre femmes particulières, CarnièresMorlanwelz, Lansman, 1997.

__, Prophètes sans dieu, Carnières-Morlanwelz, Lansman, 2003 [1999].

_ 2001.

—_, Mémoires à la dérive, Carnières-Morlanwelz, Lansman, 2004.

—_ La spirale de l'anneau, Noir Hamlet, Sacrée famille, Éditions Apopsix 2016.

__, "Traduire la réalité d'une langue à une autre ", Traduire, Genèse du choix,

Chiara Montini, éd., Paris, Éditions des archives contemporaines, 2016, p. 39-47.

—, «L'Histoire d'un exilé de l'histoire » dans Chikhi Beïda et Quaghebeur Marc (éd.), Les écrivains francophones interprètes de l'Histoire: Entre filiation et dissidence, Peter Lang, 2006, p. 261-289.

_- «Slimane Benaïssa », Interview avec Thierry Guichard, Le Matricule des Anges 44, mai-juillet 2003, p. 14-23.

—, «Au-delà du tragique: une traversée théâtrale: Entretien avec Slimane Benaïssa », par Janice Gross, French Review, vol. 87, n 4, mai 2014, p. 153-169. Cheniki Ahmed, Le théâtre en Algérie: Histoire et enjeux, Aix-en-Provence, Édisud, 2002.

Chikhi Beïda, Destinées voyageuses: la Patrie, la France, le Monde, Paris, 2006.

De Certeau Michel, Giard Luce et Mayol Pierre, L'invention du quotidien, Paris, Gallimard, Collection Folio/essais, nouvelle éd. vol. 146, 238, 1990.

Cooke Miriam, Women Claim Islam: Creating Islamic Feminism Through Literature, New York et Londres, Routledge, 2001.

De Godot à Zucco: anthologie des auteurs dramatiques de langue française, vol. 3, Paris, Éditions THÉÂTRALES, 2004.

Conquergood Dwight, «Performing as a Moral Act », Cultural Struggles, Ann Arbor, University of Michigan Press, 2013, p. 65-80.

Déjeux Jean, « Pour qui sont frappés les trois coups? » Autrement, n 38, mars 1982, p. 278-280.

Gross Janice, «Performing Beyond Trauma: Stages of Recovery in Slimane Benaïssa's Theatre », dans El Nossery Névine et Hubbell Amy L. (dir.), The Unspeakable: Representations of Trauma in Francophone Literature and Art, Newcastle upon Tyne, Cambridge Scholars Publishing, 2013, p. 73-93.

— and Mohamed Kacimi ", French Review 83.6, 2010, p. 1258-1271.

- "The Tragedy of Algeria: Slimane Benaïssa's Drama of Terrorism », Theatre Journal 54, 2002, p. 369-387. 
Au-delà du voile de Slimane Benaïssa : de la mise en parole arabe à la mise en scène occidentale

Mebarek Walid, "Slimane Benaïssa (Dramaturge): Aller à la rencontre de l'autre », El-Watan, 27-08-2008.

—, https://www.dzairnews.com/articles/elwatan-festival-d-avignon-unetete-de-pont-universelle 16-07-2008.

Milkovitch-Rioux Catherine, Mémoire vive d'Algérie, Paris, Libella, 2012.

Pavis Patrice, L'analyse des spectacles, Paris, Éditions Nathan, 1996.

- Dictionnaire de la performance et du théatre contemporain, Paris, Armand Colin, 2014.

Pretceille Martine, "Linterculturel comme paradigme de transgression par rapport au culturalisme » Voix plurielles, 12.2, 2015, p. 251-263.

Renaud Agnès, http://www.compagnie-espritdelaforge.com/compagnie/ agnes-renaud/

—_ « Présentation », http://www.theatre-contemporain.net/spectacles/ Au-dela-du-voile/ensavoirplus/

Ricœur Paul, Sur la traduction, Paris, Bayard, 2004.

Rokem Freddie, Performing History: Theatrical Representations of the Past in Contemporary Theatre, Iowa City, University of Iowa Press, 2000.

Taylor Diana, The Archive and the Repertoire: Performing Cultural Memory in the Americas, Durham, Duke University Press, 2003.

\section{Notes}

1. Retour à la scène algérienne en 2011, le monologue-mémoire Moudja wel wellat [Le retour de la vague], annonce la suite de l'éponyme Babour ghraq (1982).

2. «Au-delà du tragique », Entretien avec Benaïssa (Gross), 159.

3. Cité dans De Godot à Zucco, p. 21 (Bond tiré de « Le sens du désastre » Registres, $\mathrm{n}^{\circ}$ 6, 2001, p. 146).

4. Références tirées de $A u$-delà du voile, Lansman, 2008.

5. Jeu féminin et poétique d'un rituel de divination pour les jeunes filles.

6. Milkovitch-Rioux, 76 .

7. Chikhi, Destinées voyageuses: la Patrie, la France, le Monde, 7.

8. Voir Baffet «Entre théâtre et roman ». Seul ouvrage traduit: Dernière nuit d'un damné (Plon 2002) en [Last Night of a Damned Soul par Janice Gross et Daniel Gross, New York, Grove Press, 2004].

9. Avec Fatoum Ousliha et Dalila Halilou; mise en scène de Slimane Benaïssa.

10. Myriam Loucif et Khadija El Mahdi.

11. Rappel de Babour ghraq [Le bateau coule], pièce de Benaïssa, source des chants de révolte pendant le " premier » printemps arabe en Algérie, octobre 1988.

12. " to bring self and other together so that they can question, debate and challenge each other. It is the kind of performance that resists conclusions [...] It brings self and other together, even as it holds them apart. It is more like a hyphen than a period». (75) 
13. Grâce à l'enregistrement vidéo du spectacle algérien sur ENTV en 1991 et à l'assistance à la pièce au Lucernaire en 2011, j'ai pu observer les deux productions et leur réception auprès de divers spectateurs.

14. Pavis, Analyse, 258-260.

15. Baffet, Tradition, 184.

16. Voir Balibar « Doubles contraintes », 21-37.

17. Mebarek «Aller à la rencontre de l'autre ».

18. Renaud: http://www.compagnie-espritdelaforge.com/compagnie/agnes-renaud/

19. "Traduire la réalité d'une langue à l'autre », 42.

20. Voir « Histoire d'un exilé de l'Histoire » et «Traduire la réalité... ».

21. "reprocher à un metteur en scène de ne pas donner une représentation authentique [...], c'est toujours lui faire un mauvais procès, c'est l'accuser de ne pas être ce qu'il n'est pas » (Pavis, Dictionnaire 31).

22. Fatima Aibout.

23. Mebarek, 27-08-2008.

24. Mebarek 16-07-2008.

25. Taylor, 20.

26. Rokem, 13.

27. Pretceille, Voix plurielles, 257.

28. Marianne et le marabout (atelier avec les jeunes issus de l'immigration en banlieue, 1995); Un homme ordinaire pour quatre femmes particulières (atelier avec des femmes abusées, 1997, puis 2004) et Sacrée famille (création collective sur l'expérience immigrée des femmes de Massy, 2016). 